\title{
Impacto da Gestão para Resultados nos Indicadores Criminais do Ceará
}

\author{
Impact of Management for Results on Criminal Indicators in Ceará
}

\author{
José Raimundo de Araújo Carvalho Júnior ${ }^{1}$ \\ Rodolfo Ferreira Ribeiro da Costa ${ }^{2}$ \\ Frederico Augusto Gomes Alencar ${ }^{3}$ \\ Tiago Monteiro da Silva ${ }^{4}$
}

\begin{abstract}
Resumo
Busca-se identificar se a Lei estadual n. ${ }^{0}$ 15.558/2014 é capaz de influenciar os indicadores de criminalidade dos municípios cearenses. A análise baseiase num painel de dados para todos os 184 municípios cearenses, considerando de 2010 a 2016 . Os determinantes da criminalidade são analisados a partir do método de mínimos quadrados generalizados factíveis. Embora a expectativa prevista na Lei $n .^{\circ} 15.558 / 2014$ seja de influenciar negativamente os indicadores criminais do Ceará, as estimativas indicam que tal resultado não foi alcançado (exceto para os crimes de furto) e que essa perspectiva não é uma singularidade cearense, já que o mesmo fato pode ser observado por outras experiências brasileiras e internacionais. Por possibilitar ganhos financeiros diretos, tal mecanismo pode ser responsável por elevar o esforço na segurança local, que passa a atuar de forma mais intensa e, consequentemente, a registrar um maior número de ocorrências. Contudo o incentivo financeiro não se mostra tão impactante para o combate da criminalidade, devendo esse fenômeno ser melhor estudado, considerando outras abordagens, quais sejam: comparar o Ceará com outro estado, comparar o Ceará com uma média de unidades federativas e comparar os municípios do CE antes e depois da Gestão para Resultados (GPR) para avaliar o efeito da lei.
\end{abstract}

Palavras-chave: criminalidade. gestão para resultados. segurança pública.

\begin{abstract}
It seeks to identify if State Law No. 15,558/2014 is capable of influencing the crime indicators of Ceará municipalities. The analysis is based on a panel of data for all 184 cities in Ceará, considering from 2010 to 2016. The determinants of crime are analyzed using the feasible generalized least squares method. Although the expectation foreseen in Law 15.558/2014 is to negatively influence the criminal indicators of Ceará, the estimates indicate that this result was not achieved (except for theft crimes) and that this perspective is not a singularity in Ceará, since that the same fact can be observed by other Brazilian and international experiences. As it enables direct financial gains, this mechanism may be responsible for increasing the effort in local security, which starts to act more intensely and, consequently, to register a higher number of occurrences. Still, the financial incentive is not so impactful to fight crime, and this phenomenon should be better studied, considering other approaches, namely: comparing Ceará with another state, comparing Ceará with an average of federative units, and comparing municipalities in Ceará before and after the Management for Results (GPR) to assess the effect of the law.
\end{abstract}

Keywords: criminality. management for results. public security.

Ph.D. em Economia - Pennsylvania State University/USA (2002). Professor Titular da Universidade Federal do Ceará do Curso de Pós-Graduação em Economi. Pesquisador nivel 2 do CNPq. Doutorado em Economia pela Universidade Federal do Ceará (2013) e Pós Doutorado pela Universidade Federal do Rio Grande do Norte (2015). Professor Adjunto da Universidade do Estado do Rio Grande do Norte e Coordenador do Programa de Pós Graduação em Economia. Engenheiro Aeronáutico pelo Instituto Tecnológico de Aeronáutica. Mestrado em Economia pela Universidade Federal do Ceará, Mestrado em Estatística pela University of Wisconsin-Madison. E, Doutorado e Mestrado em Economia pela University of Wisconsin-Madison. 


\section{Introdução}

O Brasil atingiu um patamar de criminalidade nunca antes visto. De acordo com o Fórum Brasileiro de Segurança Pública (2017), 61.619 pessoas foram assassinadas de maneira violenta em 2016 (sete pessoas mortas por hora), o maior número já registrado pelo estudo em uma década. Tal magnitude, para se ter dimensão, equivale ao total de vítimas da explosão da bomba atômica em Nagasaki, no Japão, em 1945. Segundo a Fundação Instituto de Pesquisa Econômica Aplicada e o Fórum Brasileiro de Segurança Pública (2017), houve uma tendência de aumento da violência a partir de 2007, seja na quantidade de homicídios, seja na taxa de homicídios a cada 100 mil habitantes. Ainda, o projeto Monitor da Violência do G1 constatou que 64\% dos casos denunciados (1.195 mortes violentas ocorridas em todo o país entre 21 e 27 de agosto de 2017, uma a cada oito minutos) continuam com as investigações em aberto. Tal fato demonstra precariedade nas investigações, resultando em impunidade aos criminosos.

Considerando agora uma abordagem local, de acordo com o relatório "Diagnóstico dos homicídios no Brasil: subsídios para o Pacto Nacional pela Redução de Homicídios”, elaborado pelo Ministério da Justiça em 2015, o Ceará apresentou, em 2014, a maior taxa de homicídios a cada 100 mil habitantes do Brasil $(46,9)$, sendo comparável àquelas de países com histórico de guerras civis, como o Congo $(30,8)$, ou países com envolvimento em narcotráfico, como a Colômbia $(33,4)$. Diante desse cenário, de que forma o Estado brasileiro poderia atuar para suprir a demanda da sociedade por melhor prestação de serviços na segurança pública, especialmente no Ceará? Para Holanda e Rosa (2004), a gestão para resultado (GPR), um modelo em que o setor público passa a adotar uma postura empreendedora, voltada para o cidadão como cliente e buscando padrões ótimos de eficiência, eficácia e efetividade, com ética e transparência, seria uma solução plausível. A GPR propõe um gerenciamento otimizado dos recursos gastos por meio de indicadores e um alinhamento com os objetivos estratégicos de uma organização.

Em 2014, por meio da Lei n. ${ }^{\circ} 15.558$, de 11/03/2014, visando empreender esforços contrários aos relatos de crescimento da atividade criminosa, o estado do Ceará, por meio de sua Secretaria de Segurança Pública e de Defesa Social (SSPDS), implanta um esquema de incentivos com o objetivo de reduzir os indicadores de criminalidade. A partir desse advento, questiona-se como a efetivação da Lei n. ${ }^{\circ}$ 15.558/2014 pode viabilizar o modelo de GPR. Qual o efeito prático da aplicação da referida norma? Pretende-se, pois, de maneira geral, verificar quais os resultados produzidos pela implantação da Lei n. ${ }^{\circ} 15.558 / 2014$ no âmbito da segurança pública do Ceará. Para tanto, foi construído um painel de dados para todos os 184 municípios cearenses, considerando de 2010 a 2016 . A estratégia empírica baseia-se na estimação da função de criminalidade, baseada na proposta de Becker (1968), por meio do método de mínimos quadrados generalizados factíveis. Os indicadores de criminalidade utilizados foram crimes violentos, letais e intencionais (CVLI), crimes violentos contra o patrimônio (CVP), apreensão de armas (AA), apreensão de entorpecentes $(A E)$ e furtos. Espera-se que a referida proposta seja capaz de influenciar e propor melhorias no sistema de premiação por resultados adotados pela SSPDS.

Além dessa seção introdutória, este trabalho está dividido em mais duas seções. A segunda parte descreve a política pública implantada pelo governo cearense na área de segurança pública por meio da Lei n. ${ }^{\circ} 15.558 / 2014$, possuindo uma subseção que aborda a metodologia utilizada, a análise dos dados em painel, o modelo empírico e a descrição da base de dados utilizada, além de outra subseção com a demonstração de resultados do impacto da implantação de GPR na área de segurança pública cearense, a partir de testes de hipóteses e realização de estimativas. Finalmente, a terceira seção aborda conclusões acerca dos resultados encontrados, no sentido de contribuir com a redução da criminalidade no Ceará.

\section{Desenvolvimento}

É desejável que o Estado brasileiro assimile novos conceitos, seja do ambiente privado, seja de exemplos de sucesso da sua própria administração, via contratação de consultorias ou realização de benchmarking (palestras, congressos, divulgação de boas práticas de governos etc.). Essa prática é uma necessidade da máquina pública para se reinventar frente às grandes demandas do cidadão por serviços públicos. O bem-estar do cidadão é para ser a razão maior da existência e atuação do Estado brasileiro. Nesse sentido, a GPR é uma política de gerenciamento bem aceita, pois proporciona, como eixo principal de atuação, o impacto na ponta dos processos, ou seja, na população.

A empresa de consultoria denominada Macroplan, contratada em duas ocasiões (2003 e 2006) pelo Governo do Ceará, assevera que a gestão estratégica orientada para resultados transforma intenções estratégicas em resultados concretos. A referida contratação de consultoria para implantação da GPR no estado do Ceará não seria suficiente se não fosse acompanhada da criação de mecanismos de disseminação e implantação da nova cultura e do fortalecimento de governança.

$\mathrm{Na}$ área de segurança pública cearense, observa-se o Sistema de Compensação pelo Cumprimento de Metas por Indicadores Estratégicos de Criminalidade no Estado do Ceará, objeto de estudo da presente pesquisa e que possui o arcabouço normativo descrito na "Tabela 1. Cabe acrescentar que essa mudança de paradigma na forma de remuneração das forças policiais teve forte influência de Dantas (2014). Para a implementação de sistemas de compensação, é necessário um rigoroso, detalhado e criterioso processo de avaliação técnico-científica, já que existe 
a possiblidade da ação de incentivos perversos, que induzem ao moral hazard, desrespeito aos direitos humanos, desencorajamento de colaboração e cooperação.

Tabela 1 - Legislação relacionada com a GPR implantada na segurança pública cearense.

\author{
Portaria n. ${ }^{\circ}$ 1.677/2013-GS, de 01/10/2013 (DOE 11/10/13); \\ Lei n. ${ }^{\circ} 15.558$, de 11/03/2014 (DOE 18/03/2014); \\ Lei Complementar n. ${ }^{\circ} 133$, de 11/03/2014 (DOE 18/03/2014); \\ Decreto n. ${ }^{\circ} 31.448 / 2014$, de 24/03/2014 (DOE 31/03/2014); \\ Portaria n. ${ }^{\circ}$ 090/2014-GS, de 13/03/2014 (DOE 31/03/2014); \\ Portaria n. ${ }^{\circ} 436 / 2014-G S$, de 02/01/2014 (DOE 31/03/2014); \\ Decreto . $^{\circ} 31.496$, de 23 de junho de 2014, de 23/06/2014 (DOE 24/06/14); \\ Portaria Normativa n. ${ }^{\circ}$ 436/2014-GS, de 11/04/2017 (DOE 17/04/2017); \\ Acordo de Resultados n. ${ }^{\circ}$ 01/2017, de julho de 2017 (GOVERNADOR, SEPLAG e SSPDS.
}

Fonte: Elaboração própria.

Em síntese, o modelo aplicado remunera as forças policiais (Polícia Militar, Polícia Civil, Perícia Forense e Corpo de Bombeiros) proporcionalmente ao atingimento de indicadores de desempenho de meta (salvar vidas, proteger pessoas e patrimônio, solução de crimes e prisões, vistorias técnicas e afogamentos) previamente determinados. A compensação pecuniária também é influenciada pelos seguintes aspectos: localização geográfica do servidor público; desempenho de outras Áreas Integradas de Segurança (AIS), dos territórios e do estado; patente do policial (mais responsabilidades); peso que uma dada meta possui; dias trabalhados e valor residual (VR). O pagamento do VR será distribuído, por ordem de classificação, para as 15 melhores AIS em produtividade absoluta, relativamente a sua respectiva contribuição à meta do estado, respeitando o limite definido da $1^{\circ}$ colocada receber o VR 10 vezes maior que a $15^{\circ}$ de forma linear. Vale salientar que cada AIS possui um agrupamento de forças policiais (Batalhão PM, Distrito Policial, Delegacias Metropolitana, Seccional, Regional ou Municipal, Plantonista e CB) responsável por cada área.

Várias são as estatísticas criminais gerenciadas pela SSPDS, sendo que o CVLI é o indicador mais propício para representar a criminalidade, pois se traduz na violência mais extrema e um atentado a vida em sociedade, além de ser o mais usado internacionalmente para medir a violência. Influencia também o fato de que os outros tipos de crimes possuem taxas menores de registros pelas vítimas. Segundo Kassouf e Santos (2008), pressupõe-se que as tendências da criminalidade sejam bem representadas pelas tendências dos homicídios e que há um melhor ajuste para modelos que utilizam crimes letais contra a pessoa como variável dependente. Isso se deve ao fato de que a taxa de sub-registro nesse tipo de crime é significativamente pequena por implicar perda de vida humana e, consequentemente, registro no Instituto Médico Legal.

A GPR é uma ação em que os governos tentam dar uma resposta às crescentes demandas sociais por mais e melhores serviços públicos prestados. A partir dessa contextualização, será possível definir uma metodologia e método econométrico para avaliar especificadamente o impacto da política pública representada pela Lei $\mathrm{n}$. 15.558/2014 na segurança pública do Ceará.

\title{
2.1 Aspectos metodológicos e seleção de modelos
}

A análise dos determinantes da criminalidade, conforme Becker (1968), pode ser realizada a partir da decisão do indivíduo entre cometer ou não a ação criminosa. Para tanto, o tomador de decisão afere, a partir das suas expectativas quanto aos benefícios e aos custos oriundos do delito, o resultado dessa ação e se existem, de fato, incentivos para agir de forma dolosa.

Embora, teoricamente, a tomada de decisão do agente que comete um crime possa ser definida pela expectativa do resultado esperado, a estratégia empírica para uma função de criminalidade baseia-se em elementos que podem ser associados a tais expectativas. Nesse sentido, um conjunto de proxy's é utilizado para retratar componentes referentes aos benefícios e custos associados à atividade criminosa, entre eles: a renda, a densidade demográfica, a pobreza, a educação, os gastos com segurança etc. Essa metodologia foi utilizada em diversas pesquisas, como em Loureiro e Carvalho (2007), Kassouf e Santos (2008), Fernandes Jr et al (2017) e Fernandez e Pereira (2000).

Desse modo, para identificar os determinantes da criminalidade no estado do Ceará e, ainda, se a Lei n. ${ }^{\circ}$ 15.558/2014, que afeta diretamente a ação policial com mecanismos de incentivo, bem como outros delitos, foi capaz de influenciar a ação definida como dolosa, este trabalho buscou estimar funções de criminalidade. Para tanto, as construções das referidas funções foram conduzidas a partir de um painel de dados que englobou como variável dependente o número de delitos; como controle, proxy's que configurem os elementos de custo e benefícios da atividade; e, por fim, uma variável dummy para captar o efeito da política descrita pela Lei n. ${ }^{0}$ 15.558/2014. 
A opção pela estrutura de dados em painel justifica-se, entre outros aspectos já comentados, pelo fato da análise da Lei n. $^{\circ}$ 15.558/2014 ter sido sancionada a partir de 2014 e, portanto, para que possa se mensurar seu respectivo efeito sobre a criminalidade antes e depois da mencionada lei, faz-se necessário o uso de uma série de tempo (acompanhamento das variáveis ao longo do tempo) e, ainda, por se tratar de uma política direcionada a todos os municípios do estado do Ceará (elevado tamanho da amostra), a presente discussão deve relatar todos os elementos da amostra em questão.

Algumas vantagens do uso de dados em painel indicadas por Kassouf e Santos (2008), Fernandes Jr et al (2017) e Araujo Jr e Fajnzylbe (2001) são: minimização de eventuais problemas de colinearidade entre as variáveis explicativas e identificação da heterogeneidade e melhor condição ao processo de inferência. Assim, dadas as características da análise e o perfil das unidades envolvidas, a estrutura recomendada seria a de dados em painel.

A estrutura de dados em painel pode der definida como o acompanhamento de unidades transversais em diferentes períodos. Entre suas vantagens, a elevação do tamanho da amostra e a minimização de eventuais problemas de colinearidade entre as variáveis explicativas possibilitam ganhos essenciais a modelagem. Contudo seus principais acréscimos, relativos às estimativas com dados em cross-sections, dar-se-ão pelo fato da identificação da heterogeneidade entre as unidades e, também, pela possibilidade de seu acompanhamento ao longo do tempo, permitindo, assim, uma análise da dinâmica inerente à relação entre as variáveis de análise.

Os estimadores para dados em painel são definidos a partir de um conjunto de informações que contemplam um dado número de unidades e o tempo. É razoável que as unidades sejam as mesmas ao longo dos períodos e que observações ausentes não apresentem uma proporção significativa. De fato, a estrutura em painel requer que $i$ unidades, existentes ao longo de $t$ períodos, sejam as mesmas, tal que seu comportamento individual possa ser comparado. Ainda, a presença de missing deve ser minimizada para que se trate de um painel com um maior número de informações e, portanto, com maior número de graus de liberdade, garantindo uma maior eficiência às estimativas.

Entre os estimadores para dados em painel, destacam-se: os de efeito fixo e de efeito aleatório. Em síntese, ambos os estimadores buscam incluir na função de regressão o termo que capte a heterogeneidade entre as unidades amostrais, sendo esse considerado um valor fixo ou não ao longo do tempo, embora possam ter valores diferentes entre as unidades, respectivamente.

Formalmente, o estimador de efeito fixo pode ser apresentado como segue:

$$
Y_{i t}=c_{i}+X_{i t} \beta+e_{i t}
$$

Onde $Y_{i t}$ é a variável dependente, ou aquele cujo comportamento deseja ser explicado, para a unidade $i$ no período $t ; c_{i}$ é o parâmetro que capta a heterogeneidade entre as unidades; $X_{i t}$ é a matriz de variáveis explicativas da unidade $i$ no período $t ; \beta$ é o vetor de parâmetros que captam as mudanças em $Y_{i t}$ dada variações em $X_{i t}$; por fim, $e_{i t}$ é o termo de erro.

Wooldridge (2002) comenta que a vantagem do estimador de efeito fixo em relação ao de MQO depende da existência de uma relação entre o termo $c_{i}$ e $X_{i t}$. Caso tal comportamento seja observado, as estimativas de MQO serão enviesadas e inconsistentes, favorecendo o uso do estimador de efeito fixo.

O estimador de efeito aleatório pode ser definido admitindo que o termo que capta a heterogeneidade entre as unidades analisadas pode ser considerado como um componente aleatório. Formalmente, tal estrutura apresenta semelhança com a equação (1). Contudo o termo passa a compor o termo de erro. Assim, pode-se definir a função de regressão da qual o estimador de efeito aleatório é extraído como segue:

$$
\text { Em que } \boldsymbol{Y}_{\boldsymbol{i t}}=\boldsymbol{X}_{\boldsymbol{i t}} \boldsymbol{\beta}+\boldsymbol{v}_{\boldsymbol{i t}}
$$

Segundo Hill, Griffiths e Judge (1999), a equação (2) deve satisfazer as seguintes condições:

(i) $\mathrm{E}\left(v_{i t}\right)=0$;

(ii) $\operatorname{var}\left(v_{i t}\right)=\sigma_{c}^{2}+\sigma_{e}^{2}$;

(iii) $\left.\operatorname{cov}\left(v_{i t}, v_{i s}\right)\right)=\sigma_{c}^{2}, \mathrm{t} \neq \mathrm{s}$;

(iv) $\operatorname{cov}\left(v_{i t}, v_{j t}\right)=0, \mathrm{i} \neq \mathrm{j}$.

As condições (i) e (ii) tratam das condições de exogeneidade e de homocedasticidade, respectivamente. A terceira e a quarta discutem a forma na qual o termo de erro apresenta correlação. A abordagem de efeito aleatório admite que haja correlação serial entre os erros de períodos distintos, não sendo o mesmo válido para o nível das unidades analisadas. Essas últimas características deixam clara a condição no qual o estimador MQO torna-se inadequado. Assim, na presença de heterogeneidade, a autocorrelação serial torna a utilização do estimador de efeito aleatório uma solução apropriada. 
Embora existam vantagens teóricas no uso dos estimadores de efeito fixo e aleatório em relação ao estimador de MQO, seus respectivos usos devem ser condicionados às questões de natureza da pesquisa e/ou aos testes de especificação. No primeiro caso, ao se tratar unidades com características distintas, é razoável admitir que as unidades amostrais possam ser heterogêneas e, portanto, a caracterização da função de regressão através das equações (1) ou (2) poderia simplesmente ser considerada. No entanto Bressan (2009) destaca que um conjunto de testes de especificação podem ser utilizados para definição mais adequada do estimador a ser utilizado. A estratégia requer, inicialmente, a decisão entre o estimador de MQO e os estimadores de efeito fixo e aleatório, definidos pelo teste de Chow e Breusch-Pagan, respectivamente. Em seguida, caso as opções de efeito fixo e aleatório sejam escolhidas, realiza-se o teste de Hausman, definido sob a hipótese nula de que não haja diferença entre os estimadores. Finalmente, são testadas as condições de erro esférico e, se confirmada, a opção recomendada seria o uso do estimador de mínimos quadrados generalizados factíveis.

Para analisar os determinantes do crime no estado do Ceará e, ainda, identificar se a Lei n. ${ }^{\circ} 15.558 / 2014$ foi capaz de influenciar a trajetória da criminalidade, este trabalho considerou elementos socioeconômicos, políticos e comportamentais que possam ser considerados determinantes no processo de decisão da atividade dolosa. Assim, admitindo que tais elementos podem refletir os benefícios e custos associados à criminalidade e, assim, definir a expectativa de resultado do criminoso, é possível definir as funções de criminalidade como segue:

$$
\begin{gathered}
\text { crime }_{i t}=\beta_{0}+\beta_{1} \text { leigpr }_{t}+\beta_{2} \text { bpraio }_{t}+\beta_{3} \text { escol }_{i t}+\beta_{4} \text { densidade }_{i t}+ \\
\beta_{5} \text { emp }_{-} \text {saldo }_{i t}+\beta_{6} \text { gastos }_{i t}+\beta_{7} \text { fundeb }_{i t}+\beta_{8} \text { icms }_{i t}+ \\
+\beta_{9} \text { ipva }_{i t}+\beta_{10} \text { royalties }_{i t}+\beta_{11} \text { ip }_{i t}+\beta_{12} \text { itr }_{i t}+v_{i t}
\end{gathered}
$$

Em que crime $_{i t}$ é o número de crimes da unidade $i$ no período $t$, sendo utilizados como medida os crimes violentos letais e intencionais (CVLI), crimes violentos contra o patrimônio (CVP), apreensão de armas (AA), apreensão de entorpecentes (AE) e furtos (Furto); leigpr $t_{t}$ é uma variável dummy que assume valor 1 a partir do ano de sanção da lei e zero para os demais anos; bpraio $_{t}$ é uma variável dummy que assume valor 1 quando a unidade tem BPRaio e valor zero caso contrário; escol ${ }_{i t}$ é o índice desempenho escolar da unidade $i$ no período $t$; densidade $\boldsymbol{i}_{i t}$ é a densidade demográfica da unidade $i$ no período $t$; emp_saldo $i t$ é a diferença do número de postos de trabalho criados e destruídos a unidade $i$ no período $t$; gastos $i$ são os gastos em assistência social (valor dos benefícios emitidos pela Previdência Social) da unidade $i$ no período $t$; funde $b_{i t}$ são as transferências do Fundeb recebidas pela unidade $i$ no período $t$; icms it $_{i t}$ é a arrecadação de ICMS da unidade $i$ no período $t$; ipva ${ }_{i t}$ é a arrecadação de IPVA da

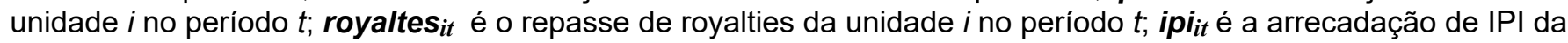
unidade $i$ no período $t$; itr $i$ é a arrecadação de ITR da unidade $i$ no período $t$; os $\boldsymbol{\beta}_{i}$ 's são os parâmetros estimados; por fim, $\boldsymbol{v}_{\text {it }}$ é o termo de erro aleatório.

A unidade $i$ refere-se aos municípios cearenses que, atualmente, são 184. Ocorre que existe uma relação entre os municípios e as respectivas AIS, de modo que o município de Fortaleza, que tem grande concentração demográfica, é dividido em 10 AIS, conforme seus bairros. Considerando que as metas previstas pela Lei $n .^{\circ} 15.558 / 2014$ são estipuladas e avaliadas a partir de cada AIS, foi necessário quantificar as unidades i em 193, de tal maneira que Fortaleza fosse dividida em consonância com suas respectivas AIS (AIS de 01 a 10). Assim, as AIS de 01 a 10 possuem as variáveis explicativas referentes ao município de Fortaleza e as respectivas variáveis dependentes específicas de suas AIS.

Foram levantadas 75 variáveis relacionadas ao crime, das quais 23 foram classificadas como inelegíveis (seja pelo intervalo de anos inadequado, seja pela abrangência nacional ou estadual) e 52 foram classificadas como elegíveis. Dessas válidas, apenas 13 variáveis foram utilizadas por serem mais significativas. A partir desse levantamento de variáveis, verificou-se que o período de 2010 a 2016 foi o intervalo que continha a maior amostra possível para formar um painel de dados balanceado. A base de dados originalmente constituída em uma planilha eletrônica contém 57 colunas e 1.352 linhas e foi, posteriormente, carregada no software STATA/MP, versão 14.0, para as devidas estimações. Os dados utilizados na estimação da equação (3) remetem às informações sobre as 193 unidades $i$ de 2010 a 2016, nas categorias de segurança, demografia, educação, renda social e transferências constitucionais. Suas respectivas definições, fontes e efeitos esperados podem ser observados na Tabela 2. 
Tabela 2 - Descrição das variáveis, fonte e efeitos esperados.

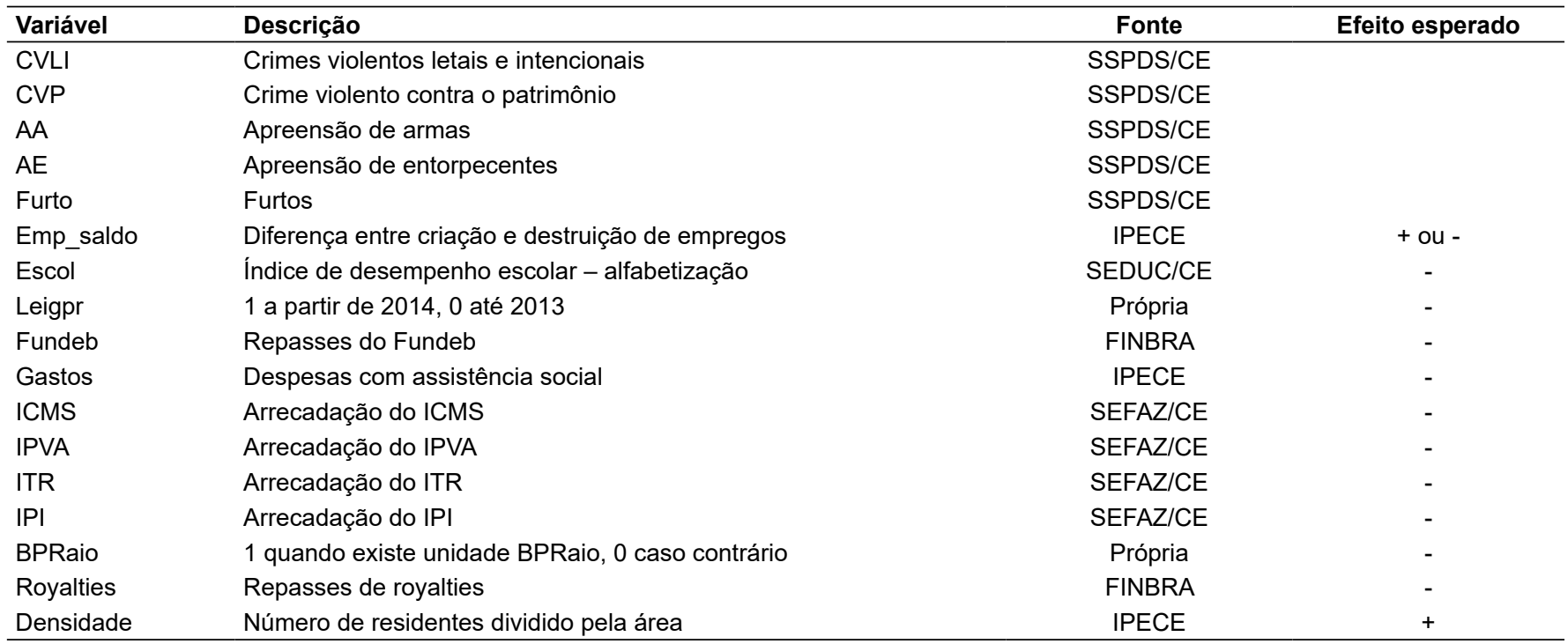

Fonte: elaboração própria.

\subsection{Resultados}

Visando captar os efeitos da gestão para resultados, idealizada pela Lei . $^{\circ} 15.558 / 2014$, sobre a segurança no estado do Ceará e, assim, identificar se tal proposição é capaz de influenciar o indicador do CVLI, entre outros indicadores, e, em caso positivo, qual a forma como a provocação se processa, este trabalho estima cinco funções de criminalidade baseadas na proposta de Becker (1968). Nesse sentido, buscou-se, por meio de um conjunto de variáveis socioeconômicas, das contas públicas e de uma dummy para a referida lei, explorar os fenômenos que gerem a decisão do indivíduo na idealização da prática criminosa.

De forma mais específica, a construção da função de criminalidade permitirá o entendimento de como a prática criminosa pode ser influenciada pela política de gestão para resultados, ou seja, de como o mecanismo de incentivo criado por essa última é capaz de modificar a decisão do agente sobre cometer o crime. Segundo Becker (1968), é razoável supor que, se um conjunto de variáveis influenciam negativamente o resultado esperado do crime, então são capazes de reduzir o número de crimes. Assim, diante do propósito da Lei n. ${ }^{\circ} 15.558 / 2014$, espera-se que a mesma pertença ao grupo de elementos inibidores do crime e, portanto, haja de forma negativa sobre a criminalidade ocorrida no estado do Ceará.

Os resultados das estimações, obtidas a partir da equação (3) e dos dados apresentados na seção 2.2, seguem a rotina clássica definida para escolha entre as estruturas pooled e de dados em painel. Primeiramente, são testadas as hipóteses nulas de modelo pooled contra os estimadores de efeito fixo e de efeito aleatório, por meio dos testes de Chow e de Breusch-Pagan, respectivamente ("Tabela 3).

Tabela 3 - Testes: pooled $\mathrm{x}$ dados em painel.

\begin{tabular}{llccc}
\hline Modelo & Teste & Hipótese nula & Estatística & $\boldsymbol{p}$-valor \\
\hline \multirow{2}{*}{ CVLI } & Chow & Pooled & 33.67 & 0.000 \\
& Breusch-Pagan & Pooled & 892.26 & 0.000 \\
CVP & Chow & Pooled & 7.49 & 0.000 \\
& Breusch-Pagan & Pooled & 322.40 & 0.000 \\
AA & Chow & Pooled & 49.87 & 0.000 \\
& Breusch-Pagan & Pooled & 964.09 & 0.000 \\
AE & Chow & Pooled & 2.02 & 0.000 \\
\multirow{2}{*}{ Furto } & Breusch-Pagan & Pooled & 45.49 & 0.000 \\
& Chow & Pooled & 17.07 & 0.000 \\
\end{tabular}

Fonte: elaboração própria. 
Dos resultados da Tabela 3, é possível definir que a estrutura pooled se mostra inferior aos dados em painel. Tal fato, inicialmente, reflete que haja heterogeneidade entre as unidades observadas; segundo, tal indicação corrobora para uma inibição de possíveis problemas de colinearidade; terceiro, diante de uma estrutura com dados em painel, um maior volume de observações é considerado, elevando os graus de liberdade e, assim, garantindo uma melhor condição ao processo inferência.

Seguindo, ao admitir uma estrutura com dados em painel, é razoável identificar qual a forma que melhor caracteriza a heterogeneidade das unidades. A indicação que melhor representaria tal condição pode ser obtida por meio do teste de Hausman, o qual transita entre a escolha por um estimador de efeito aleatório ou de efeito fixo. Finalmente, admitindo a hipótese de exogeneidade estrita, resta observar se o termo de erro se caracteriza como do tipo esférico, em outras palavras, seja homocedástico e não seja autocorrelacionado. Os resultados de tais requisitos estão expressos na Tabela 4.

Tabela 4 - Teste de Hausman, Wooldridge e Wald.

\begin{tabular}{|c|c|c|c|c|}
\hline Modelo & Teste & Hipótese Nula & Estatística & p-valor \\
\hline & Hausman & Efeito aleatório & 15.74 & 0.003 \\
\hline \multirow[t]{3}{*}{ CVLI } & Wooldridge & Não há autocorrelação & 0.321 & 0.572 \\
\hline & Wald & Homocedasticidade & $15,134.32$ & 0.000 \\
\hline & Hausman & Efeito aleatório & 273.32 & 0.000 \\
\hline \multirow[t]{2}{*}{ CVP } & Wooldridge & Não há autocorrelação & 5.07 & 0.025 \\
\hline & Wald & Homocedasticidade & $1.2 e+05$ & 0.000 \\
\hline \multirow[t]{3}{*}{ AA } & Wooldridge & Não há autocorrelação & 1.09 & 0.297 \\
\hline & Wald & Homocedasticidade & $7,473.69$ & 0.000 \\
\hline & Hausman & Efeito aleatório & 3.18 & 0.528 \\
\hline \multirow[t]{3}{*}{$A E$} & Wooldridge & Não há autocorrelação & 6.00 & 0.015 \\
\hline & Wald & Homocedasticidade & $3.9 e+07$ & 0.000 \\
\hline & Hausman & Efeito aleatório & 36.05 & 0.000 \\
\hline \multirow[t]{2}{*}{ Furto } & Wooldridge & Não há autocorrelação & 1.52 & 0.219 \\
\hline & Wald & Homocedasticidade & $62,255.76$ & 0.000 \\
\hline
\end{tabular}

Fonte: elaboração própria.

Os resultados da Tabela retratam a não aceitação da hipótese nula pelo teste de Hausman e, assim, definem como melhor opção o estimador de efeito fixo, salvo para o modelo da variável dependente (ae). Essa indicação reforça a propriedade de consistência do estimador de efeito fixo. Ainda, de acordo com a estatística de Wald, a proposição de um erro esférico é violada. Embora o teste de Wooldridge tenha aceitado a hipótese de um erro não autocorrelacionado, a estatística de Wald indica a composição de um erro heterocedástico e, portanto, requer uma medida de correção ao estimador.

Nesse sentido, a correção das estimativas pode ser executada a partir da estimação robusta, do estimador de bootstrap, do processo de jacknife ou, ainda, pelo estimador de mínimos quadrados generalizados factíveis (MQGF). Diante dessas opções, optou-se pelo estimador MQGF, devido ao melhor ajuste do modelo. A Tabela 5 apresenta as estimativas do modelo de criminalidade obtidas pelo estimador de FGLS.

Analisando os resultados da "Tabela 5, é possível verificar que as variáveis explicativas afetam o comportamento das variáveis de criminalidade ao nível de $1 \%$ de significância, com ressalva feita para alguns casos específicos, em que as variáveis de receitas e gastos não foram significativas. Tal ajustamento em relação às variáveis explicativas permite categorizar os efeitos observados sobre um conjunto de variáveis de criminalidades e, assim, inferir não somente sobre o efeito da Lei . $^{\circ} 15.558 / 2014$ sobre o CVLI, mas também sobre outras modalidades de delitos. Além disso, a observação dos efeitos marginais pode sinalizar um componente adicional aos efeitos sobre a criminalidade: qualquer que seja a ação inicialmente tomada para reduzir o número de CVLI, traz como reflexo um spillover sobre outros atos ilícitos, como apreensão de armas e entorpecentes, furtos e crimes contra o patrimônio. 
Tabela 5 - Estimativas do modelo de criminalidade

\begin{tabular}{|c|c|c|c|c|c|}
\hline \multicolumn{6}{|c|}{ Modelo } \\
\hline & cvli & cvp & aa & ae & Furto \\
\hline LEIGPR & $\begin{array}{l}15.2937^{*} \\
(2.9170)\end{array}$ & $\begin{array}{l}83.7402^{*} \\
(33.7298)\end{array}$ & $\begin{array}{l}59.9517^{*} \\
(4.2372)\end{array}$ & $\begin{array}{l}6.4952^{\star} \\
(2.7795)\end{array}$ & $\begin{array}{c}-309.1785^{*} \\
(41.9618)\end{array}$ \\
\hline BPRaio & $\begin{array}{l}36.9249^{*} \\
(6.0810)\end{array}$ & $\begin{array}{c}382.9325^{*} \\
(127.6151)\end{array}$ & $\begin{array}{l}-445.697^{*} \\
(12.6000)\end{array}$ & $\begin{array}{c}-9.4616 \\
(14.8205)\end{array}$ & $\begin{array}{l}179.1419^{*} \\
(60.8659)\end{array}$ \\
\hline Escolaridade & $\begin{array}{l}-0.6656^{\star} \\
(0.0761)\end{array}$ & $\begin{array}{c}-11.8659^{*} \\
(1.7485)\end{array}$ & $\begin{array}{l}-1.1697^{*} \\
(0.2393)\end{array}$ & $\begin{array}{l}-0.9379^{*} \\
(0.2283)\end{array}$ & $\begin{array}{l}-6.4980^{*} \\
(1.6714)\end{array}$ \\
\hline Densidade & $\begin{array}{l}0.1024^{*} \\
(0.0159)\end{array}$ & $\begin{array}{c}1.9967^{*} \\
(0.3832)\end{array}$ & $\begin{array}{c}0.0353 \\
(0.0475)\end{array}$ & $\begin{array}{l}0.1213^{* *} \\
(0.0613)\end{array}$ & $\begin{array}{l}0.819{ }^{*} \\
(0.2424)\end{array}$ \\
\hline emp_saldo & $\begin{array}{l}0.0045^{*} \\
(0.0011)\end{array}$ & $\begin{array}{c}0.0215 \\
(0.0161)\end{array}$ & $\begin{array}{c}0.0087^{*} \\
(0.0023)\end{array}$ & $\begin{array}{l}0.0068 * \\
(0.0027)\end{array}$ & $\begin{array}{c}0.0286 \\
(0.0181)\end{array}$ \\
\hline Gastos & $\begin{array}{l}-0.0001 \\
(0.0001)\end{array}$ & $\begin{array}{c}0.0014 \\
(0.0014)\end{array}$ & $\begin{array}{c}0.0004^{*} \\
(0.0001)\end{array}$ & $\begin{array}{l}0.0006^{*} \\
(0.0002)\end{array}$ & $\begin{array}{l}-0.0045^{\star} \\
(0.0009)\end{array}$ \\
\hline ICMS & $\begin{array}{l}-9.29 \mathrm{e}-07^{*} \\
(2.81 \mathrm{e}-07)\end{array}$ & $\begin{array}{c}5.73 e-06 \\
(5.36 e-06)\end{array}$ & $\begin{array}{l}-7.10 e-08 \\
(6.99 e-07)\end{array}$ & $\begin{array}{l}-6.05 e-07 \\
(8.31 e-07)\end{array}$ & $\begin{array}{l}-0.00001^{*} \\
(4.13 e-06)\end{array}$ \\
\hline IPVA & $\begin{array}{c}3.93 e-09 \\
(1.63 e-06)\end{array}$ & $\begin{array}{c}-0.0002^{*} \\
(0.00004)\end{array}$ & $\begin{array}{l}-9.51 e-06 \\
(4.74 e-06)\end{array}$ & $\begin{array}{l}-0.00001^{*} \\
(6.27 e-07)\end{array}$ & $\begin{array}{c}0.0001^{*} \\
(0.00002)\end{array}$ \\
\hline Royaltes & $\begin{array}{l}5.25 e-06^{*} \\
(2.17 e-06)\end{array}$ & $\begin{array}{c}-0.0001^{*} \\
(0.00004)\end{array}$ & $\begin{array}{l}-3.73 e-06 \\
(4.13 e-06)\end{array}$ & $\begin{array}{c}-7.01 \mathrm{e}-07 \\
(6.27 \mathrm{e}-07)\end{array}$ & $\begin{array}{c}0.00002 \\
(0.00002)\end{array}$ \\
\hline IPI & $\begin{array}{c}0.0001^{*} \\
(0.00002)\end{array}$ & $\begin{array}{l}0.0009^{*} \\
(0.0003)\end{array}$ & $\begin{array}{c}0.0002^{*} \\
(0.00004)\end{array}$ & $\begin{array}{c}-0.0002^{*} \\
(0.00006)\end{array}$ & $\begin{array}{c}0.0003 \\
(0.0003)\end{array}$ \\
\hline ITR & $\begin{array}{c}0.00003 \\
(0.00005)\end{array}$ & $\begin{array}{c}0.0038^{*} \\
(0.0007)\end{array}$ & $\begin{array}{c}0.0004^{*} \\
(0.00007)\end{array}$ & $\begin{array}{c}0.00001 \\
(0.00009)\end{array}$ & $\begin{array}{c}0.0009 \\
(0.0005)\end{array}$ \\
\hline constante & $\begin{array}{l}186.787^{*} \\
(2.9882)\end{array}$ & $\begin{array}{l}766.8711^{*} \\
(59.0936)\end{array}$ & $\begin{array}{c}324.6461^{*} \\
(6.1326)\end{array}$ & $\begin{array}{l}114.5481^{*} \\
(13.5413)\end{array}$ & $\begin{array}{c}2,228.1^{*} \\
(43.1952)\end{array}$ \\
\hline
\end{tabular}

Fonte: elaboração própria.

Obs.: * significativo a $1 \%,{ }^{* *}$ significativo a $5 \%$. Erro padrão entre parênteses.

Diferentemente do que se previa, a variável BPRaio apresentou um efeito marginal positivo sobre o CVLI, o CVP e o furto. De fato, era de se esperar que a implantação de uma nova modalidade de policiamento fosse capaz de inibir a atividade criminosa. Contudo tal condição só pode ser garantida em longo prazo. Em curto prazo, um aumento do contingente policial pode ser responsável por um maior número de registros do que anteriormente, pois não seriam diagnosticados devido à limitação da área de atuação policial e/ou da velocidade das ocorrências. Assim, diante de um resultado positivo para o efeito marginal da variável BPRaio, pode ser plausível admitir que sua ação possa naturalmente reverter os números da atividade criminosa a partir do momento em que o agente criminoso observe a elevação da probabilidade de punição (BECKER, 1968). Ainda, os registros da apreensão de entorpecentes não sofrem influência da variável BPRaio. Finalmente, cabe destacar que, em relação às operações de apreensão de armas, a variável BPRaio segue a expectativa inicial, ou seja, apresenta um efeito marginal negativo, indicando que esse tipo de ferramenta é responsável por inibir tal tipo de delito.

$\mathrm{O}$ efeito marginal da escolaridade, em conformidade com a literatura, apresentou resultado negativo e significativo, refletindo o crescimento do custo de oportunidade em relação à atividade criminosa, na medida em que o agente se qualifica. Esse fato é corroborado por outros autores que analisaram a criminalidade no Brasil, entre eles Araújo Jr (2002), Loureiro e Carvalho (2007) e Fernandes Jr et al (2017). Além disso, é possível compreender o sentido negativo para a referida relação a partir dos ganhos de renda oriundos de uma maior escolaridade. $O$ maior nível de renda inibe o agente criminoso, já que, para um maior nível de renda, menor seria a necessidade de se cometer um delito em benefício de um maior resultado esperado, como visto em Mariano (2010).

A variável densidade demográfica, que apresentou um efeito marginal positivo, indica que, em locais onde há uma maior concentração de indivíduos, também são caracterizados por um maior número de delitos. Conforme Fernandes Jr. et al. (2017), locais com elevado número de indivíduos por $\mathrm{Km}^{2}$ dificultam a identificação do delito e, consequentemente, do infrator, reduzindo a probabilidade de captura. Ainda, por beneficiar o ato ilícito, as aglomerações possibilitam uma maior interação entre infratores, reduzindo o custo e potencializando a participação em atividades criminosas. Cabe ressaltar que tal influência não pode ser direcionada ao modelo de apreensão de armas, em que tal variável não se mostrou significativa. Embora a densidade demográfica seja um componente relevante para explicar a maioria das variáveis de criminalidade assumidas neste trabalho, o fato de não conter informações sobre a variável apreensão de armas, 
contrariando as expectativas, pode sinalizar algum nível de precariedade na fiscalização do porte ilegal de armas e, assim, a limitação das ações de segurança em relação a tal delito em ambientes caracterizados por grandes aglomerações.

O saldo de empregos também se mostrou capaz de afetar o comportamento do CVLI, da apreensão de armas e de entorpecentes, refletindo que a diferença entre o número de postos criados e a sua destruição eleva a quantidade de delitos. Uma possível interpretação, com base na Tabela 2 e na literatura, é que o desemprego está aumentando no período considerado. Portanto, é plausível que tal variável, em média, apresente valores negativos e, portanto, observese, entre as unidades analisadas, um número elevado de desempregados de forma a justificar o aumento dos referidos crimes. Fernandes e Fernandes (1995), Fernandez e Maldonado (1999), Fernandez e Pereira (2000) e Shikida (2010) destacam também que, para um maior desemprego, sugerido pela redução na atividade econômica em nível nacional, reduz-se a renda dos indivíduos, inibe sua participação no mercado formal e, consequentemente, reduz o custo de oportunidade ligado à atividade criminosa. No que tange aos crimes contra o patrimônio e furtos, o efeito do saldo de emprego não apresentou influência, sugerindo que, para tais delitos, esse indicador não altera o custo de oportunidade associado à atividade criminosa e, portanto, não haveria influência sobre o resultado esperado da atividade ilícita.

Um resultado contrário ao esperado, embora seu efeito marginal seja ínfimo, foi o dos repasses associados ao Fundeb. Tais recursos são destinados à manutenção e aperfeiçoamento da educação e, portanto, assim como o resultado da escolaridade, deveriam influenciar negativamente o número de delitos. Esse resultado pode sugerir que o referido efeito não ocorreria de forma contemporânea, mas sim de forma defasada, já que o repasse é destinado à educação básica e, assim, conduzirá a formação de futuros jovens e adultos. Nesse sentido, testar defasagens para a variável seria razoável, contudo, devido ao pequeno intervalo de dados disponível, essa possibilidade tornou-se inviável, já que se faria necessário mais do que seis períodos para captar o efeito dos repasses à educação básica sobre a tomada de decisão de jovens e adultos. No entanto, o que se observa para as variáveis de apreensão de armas e de entorpecentes é tido como nulo, refletindo que, pelo menos no tempo contemporâneo, os repasses direcionados à educação básica não afetam essas formas de delitos. Assim, embora se verifique a possibilidade de um efeito positivo ou nulo dos investimentos na educação, contrariando a expectativa inicial dos seus respectivos efeitos sobre o custo da atividade criminosa, d há indícios de que tal elemento não é capaz de inibir o crime em curto prazo.

Embora de forma pouco expressiva, o efeito marginal negativo da arrecadação de ICMS mostrou que o esforço do tesouro estadual é capaz de propiciar recursos mitigadores das seguintes atividades criminosas: CVLI e furtos. Por se tratar, em média, da maior fonte de recurso estadual, o ICMS propicia melhores condições de aparelhamento e fiscalização de delitos. Ainda, por se tratar de uma fonte que é base de repasse aos municípios, sendo $25 \%$ dos mesmos destinados às administrações municipais, esse elemento da receita possibilita a ampliação das atividades de governos locais, entre elas, a segurança e, portanto, teria a capacidade de inibir o comportamento ilícito.

Na mesma ótica da variável ICMS, as demais variáveis de receitas, como royalties e IPI, como fontes de recursos deveriam, a princípio, render recursos que possam, dependendo da legislação específica, ampliar os dispêndios em segurança. Contudo ambas as variáveis apresentaram um efeito positivo sobre o CVLI, apesar das suas magnitudes serem próximas de zero. Observa-se ainda o caso das variáveis IPVA e ITR, que não se mostraram significativas e, portanto, não seria responsável por alterar o comportamento do CVLI.

No que tange aos demais modelos, a função CVP também apresenta como característica três sentidos para os efeitos marginais relacionados às variáveis de receita. Inicialmente, as variáveis IPVA e royalties apresentaram coeficientes negativos, sugerindo que podem ser direcionadas ao aparelhamento do sistema de segurança e, portanto, inibem o comportamento criminoso. As variáveis IPI e ITR, de forma oposta, afetam positivamente a trajetória do crime contra o patrimônio e, finalmente, o ICMS mostrou-se não significativo. A função para o indicador de apreensão de armas indica que somente as receitas provenientes do ITR e do IPI são capazes de influenciar o ato ilícito, embora o faça de forma bastante moderada. O indicador de apreensão de entorpecentes é afetado de forma negativa pelo IPVA e IPI, sendo, ainda, observada a ausência de efeitos individuais do ICMS, royalties e ITR sobre o mesmo. Por fim, a função furto somente pode ser afetada, de forma específica, pelo ICMS e IPVA, sendo seus respectivos efeitos negativo e positivo.

As expectativas quanto às variáveis de receita, embora em alguns poucos casos tenham sido realizadas, não caracterizam, no geral, um resultado contraditório. A capacidade dessas variáveis afetarem de maneira individual cada elemento de criminalidade analisado, medida através do efeito marginal, e deixam claro que tal magnitude, quando significativa, mostra-se ínfima e, portanto, caracterizam-se como elementos que são subutilizados no combate da atividade criminosa. A formação de receitas, principalmente no que tange à gestão para resultados, deve-se caracterizar como elementochave da ação pública, por se tratar de componente base do esquema de incentivo financeiro.

Finalmente, a variável LEIGPR, responsável por informar se a implementação da Lei n. ${ }^{\circ}$ 15.558/2014 foi capaz de alterar o número de CVLI, bem como das demais medidas de criminalidade, apresentou um efeito marginal positivo e significativo, salvo para a resposta negativa em relação à variável furto. Portanto, o sistema de compensação não produziu os efeitos esperados de diminuição dos crimes, exceto para o crime de "furto". Apesar da proposta desse instrumento de política, de destinar recursos àquelas unidades que obtenham êxito em reduzir seus respectivos indicadores de criminalidade, seja o de incentivar a ampliação do sistema de segurança e, assim, inibir o comportamento ilícito, a nossa análise não revelou isso. Outros estados brasileiros que experimentaram alguma ação relacionada com sistema de compensação não foram exitosos, pois tiveram uma variação maior do que $20 \%$ na taxa de homicídio 
por 100 mil habitantes de 2010 a 2015. Considerando a Fundação Instituto de Pesquisa Econômica Aplicada e o Fórum Brasileiro de Segurança Pública (2017), as seguintes unidades da federação se enquadram nessa situação: RN $(75,50 \%)$, MA $(52,80 \%)$, GO $(37,50 \%)$ e AM $(20,30 \%)$. No entanto esse mecanismo, por possibilitar ganhos diretos, pode ser responsável por elevar o esforço na segurança local, que passa a atuar de forma mais intensa e, consequentemente, passa a registrar um maior número de autuações e ocorrências.

Ainda, por se tratar de uma dummy, essa variável acompanha se há mudança nas séries a partir de 2014, ano de implantação da Lei . $^{\circ}$ 15.558/2014 e, portanto, caso haja um crescimento superior nos indicadores a partir desse período, o efeito dessa variável admitiria um valor positivo, algo razoável dado que a taxa de criminalidade tem apresentado taxas crescentes segundo registros do mapa da violência.

No entanto, mesmo sendo uma proposta que visa reduzir a criminalidade, políticas de gestão para resultados podem levar algum tempo para refletirem uma real mudança nos indicadores ou mesmo não provocarem mudanças significativas. Tal resultado é destacado por autores como: Reis Neto e Assis (2010), ao discutirem a realidade mineira, que apontam para um cenário no qual o risco da premiação pode induzir a um comportamento oportunista e transformar o prêmio num simples $14^{\circ}$ salário; Faull (2010), que relata que a política de incentivos implementada na África do Sul leva a resultados perversos sobre as estatísticas de criminalidade; Wickramasinghe e Dharmasiri (2006), que observam uma baixa eficácia dos esquemas de incentivo monetário no Departamento de Polícia do Sri Lanka; e Tonge, Coombs e Batcheler (2009), que identificam que o mecanismo de recompensa inglês e galês reduz o esforço dos policiais de primeira linha e a qualidade da prestação de serviços de segurança.

Portanto, embora a expectativa prevista na Lei . $^{\circ} 15.558 / 2014$ seja de influenciar diretamente e de forma negativa o número de CVLI no estado do Ceará, as estimativas indicam que tal resultado não foi alcançado e que essa perspectiva não é uma singularidade cearense, já que o mesmo fato pode ser observado por outras experiências brasileiras e internacionais. Ainda, pode-se inferir que, com exceção do número de furtos, os demais registros da atividade criminosa relacionados à apreensão de armas, crimes violentos contra o patrimônio e a apreensão de entorpecentes seguem o observado para o número de CVLI e, portanto, indicam que medidas direcionadas à gestão para resultados apresentaram resultados semelhantes sobre diferentes indicadores de criminalidade.

\section{Conclusão}

Na perspectiva de minimizar o impacto oriundo da atividade criminosa, o governo do estado do Ceará, por meio da Lei n. ${ }^{\circ}$ 15.558/2014, propôs um mecanismo de incentivo baseado numa recompensa financeira, em que os elementos que compõem o aparelho de segurança pública recebem um prêmio em dinheiro no caso de atingirem as metas estabelecidas pela gestão. Esse esquema de incentivos é orientado a partir do sistema de gestão para resultados, implementado pela administração estadual.

Diante da importância da segurança individual e coletiva em meio ao crescimento observado dos indicadores de homicídios, furtos, roubos, crimes contra o patrimônio etc., tal ferramenta mostra-se como uma alternativa viável a ser implementada. Contudo sua ação individualizada pode não atender aos anseios da gestão pública e/ou da sociedade, requerendo, portanto, o diagnóstico mais qualificado dos determinantes da criminalidade.

Nesse sentido, este trabalho se propôs a reunir informações sobre os municípios cearenses no que tange aos aspectos socioeconômicos, contas públicas e, principalmente, à implantação da Lei n. ${ }^{0} 15.558 / 2014$, para construir uma função que descreve o comportamento dos indicadores de criminalidade, entre eles o CVLI. Tal construção, além de identificar componentes que determinam o nível da atividade ilícita, também é capaz de analisar como essa medida se comportou a partir da sanção realizada para a referida lei.

Entre os elementos que afetam a decisão por um ato ilícito, observa-se que condições estruturais - como o desemprego e a escolaridade; a gestão pública, principalmente na tributação; a operação policial, com as estratégias alternativas de combate ao crime; e, finalmente, os repasses oriundos do sistema de transferências, tais como royalties e Fundeb - são capazes de influenciar a atividade criminosa por alterar o resultado esperado dessa prática, como sugerido por Becker (1968).

Além disso, no que tange ao aspecto central desta pesquisa, o qual forma-se a partir da observação do efeito proposto pela política de gestão para resultados do governo do estado do Ceará, especificamente sobre a temática da segurança pública, regida pela Lei n. ${ }^{\circ} 15.558 / 2014$, não é possível observar uma consonância entre o resultado observado e a proposta de induzir negativamente o número de CVLI, bem como o de outras práticas ilícitas como crime contra o patrimônio, manuseio de armas ou entorpecentes. Tal fato, como destacado pela literatura, embora divergente do que se propunha, não carece de singularidade. Exemplos como os observados para os estados de São Paulo e Minas Gerais deixam claro que a referida política pode gerar comportamentos oportunistas e perversos às estatísticas de criminalidade $\mathrm{e}$, portanto, não atingirem os resultados esperados.

Cabe salientar que os resultados apresentados nesta pesquisa devem ser tratados com a devida cautela, dada a limitação na base de informações, principalmente no que tange o período referente à implantação da Lei $n$. 15.558/2014, que ainda pode ser tida como insipiente para computar um fenômeno estrutural como a criminalidade. Entretanto, diante das informações disponíveis nos três primeiros anos da aplicação da política de gestão para 
resultados, a temática da segurança pública mostra sintomas preocupantes e pouco animadores, dado que o número de atos ilícitos continua aumentando.

Em consonância com Assis e Neto (2012), continua sendo indispensável o estudo sobre quais resultados estão sendo obtidos pelas experiências de pagamento por desempenho em funcionamento no governo brasileiro. Nessa seara, vislumbra-se como possíveis pesquisas tanto a previsão dos indicadores criminais do Ceará para anos vindouros quanto o aprofundamento da análise da relação entre os fatores determinantes do crime e seus respectivos indicadores criminais. Outras possíveis abordagens de análise são: comparar o Ceará com outro estado, comparar o Ceará com a média das unidades federativas e comparar os municípios do Ceará antes e depois da GPR, buscando avaliar o efeito da lei.

\section{Referências}

ARAUJO JR., A. F. Raízes econômicas da criminalidade violenta no Brasil: um estudo usando micro dados e pseudopainel - 1981/1996. Revista de Economia e Administração, [S. I.], v. 1, n. 3. p. 1-34, jul./set. 2002.

ARAUJO JR, A. F.; FAJNZYLBE, P. O que causa a criminalidade violenta no Brasil? uma análise a partir do modelo econômico do crime: 1981 a 1996. Belo Horizonte: UFMG, 2001. 88 p.

ASSIS, L. O. M.; REIS NETO, M. T. Remuneração variável por desempenho no setor público: investigação das causas do fracasso e implicações para o estado brasileiro. Revista Eletrônica de Gestão Organizacional, Recife, v. 9, n. 3, p. 585-614, set./dez. 2011. Link. Acesso em: 28 set. 2017.

BECKER, G. S. Crime and punishment: an economic approach. Journal of Political Economy, [S. I.], v. 76, p. 169217, 1968.

BRESSAN, V. G. F. Seguro deposito e Moral Hazard nas cooperativas de crédito brasileiras. 2009. 371 p. Tese (Doutorado em Economia Aplicada) - Departamento de Economia Rural, Universidade Federal de Viçosa, Viçosa, 2009.

DANTAS, R. F. Segurança pública: um novo modelo de metas e premiações. Textos para discussões, Fortaleza, n. 106, jun. 2014. Link. Acesso em: 18 mar. 2017.

FAULL, Andrew. Missing the target: when measuring performance undermines police effectiveness. South African Crime Quarterly, [S. I.], v. 2010, n. 31, p. 19-25, 2010.

FERNANDES JUNIOR, L. et al. La criminalidade no Brasil: avaliação do impacto dos investimentos públicos e dos fatores socioeconômicos. Espacio abierto cuaderno venezolano de sociología, Maracaibo, v. 26, n. 2, p. 219-245, 2017.

FERNANDES, N. F. V. Criminologia integrada. São Paulo: Revista dos Tribunais, 1995.

FERNANDEZ, J. C.; MALDONADO, G. E. C. A economia do narcotráfico: uma abordagem a partir da experiência boliviana. Nova Economia. Belo Horizonte, v. 9, n. 2. p.137-173, 1999.

FERNANDEZ, J. C.; PEREIRA, R. A criminalidade na região policial da grande São Paulo sob a ótica da economia do crime. Revista Econômica do Nordeste, [S. I.], v. 31, p. 898-918, 2000.

FORUM BRASILEIRO DE SEGURANÇA PÚBLICA. Anuário brasileiro de segurança pública: 2017. São Paulo: FBSP, ano 11, 2017. Link: . Acesso em: 1 dez. 2017.

FUNDAÇÃO INSTITUTO DE PESQUISA ECONÔMICAAPLICADA; FÓRUM BRASILEIRO DE SEGURANÇA PÚBLICA. (org.) Atlas da violência. Rio de Janeiro: IPEA: FBSP, 2017. Link. Acesso em: 1 dez. 2017.

HILL, R. C.; GRIFFITHS, W. E.; JUDGE, G. G. Econometria. Tradução Alfredo A. de Farias. São Paulo: Saraiva, p. 147-291, 1999.

HOLANDA, M.; ROSA, A. L. Gestão pública por resultados na perspectiva do Estado do Ceará. Fortaleza: IPECE, 2004. 34 p. (Nota Técnica, n. 11).

KASSOUF, A. L.; SANTOS, M. J. Estudos econômicos das causas da criminalidade no Brasil: evidências e controvérsias.

Revista Economia, Brasília, v. 9, n. 2, p. 343-372, 2008. 
LOUREIRO A. O. F.; CARVALHO JUNIOR, J. R. de A. O Impacto dos gastos públicos sobre a criminalidade no Brasil. In: HERMANNS, Klaus; ARRAES, Ronaldo A. (org.). Desigualdades e políticas regionais. Fortaleza: Fundação Konrad Adenauer, 2007. p. 165-193.

MACROPLAN. Fortalecimento do modelo de gestão pública para resultados (GPR) no estado do Ceará: produto 1: levantamento de práticas de gestão pública para resultados - benchmarking GpR. Fortaleza, 2016.

MARIANO, R. S. Fatores socioeconômicos da criminalidade no Estado de São Paulo: um enfoque da economia do crime. São Paulo. 2010. Dissertação (Mestrado em Economia) - Pontifícia Universidade Católica de São Paulo, São Paulo, 2010.

REIS NETO, M. T.; ASSIS, L. O. M. Principais características do sistema de remuneração variável no choque de gestão em Minas Gerais: o acordo de resultados e o prêmio por produtividade. Gestão \& Regionalidade, São Caetano do Sul, v. 26, n. 76, 2010.

SHIKIDA, P. F. A. Considerações sobre a economia do crime no Brasil: um sumário de 10 anos de pesquisa. Economic Analysis of Law Review, [S. I.], v. 1, n. 2, p. 318-336, 2010.

TONGE, R.; COOMBS, H.; BATCHELER, M. Police and performance related pay: an exploratory study of rewarding individual performance in the police service. Journal of Finance and Management in Public Services, [S. I.], v. 8 , n. 1, p. 21-33, 2010.

WICKREMASINGHE, S. M.; DHARMASIRI, A. S. The effectiveness of monetary rewards in motivating police officer. Sri Lankan Journal of Management, [S. I.], v. 10, 2006.

WOOLDRIDGE, J. M. Econometric analysis of cross section and panel data. Cambridge: The MIT Press, 2002.

\section{Contato:}

Tiago Monteiro da Silva

E-mail: tiagomonteirods@gmail.com

Rodolfo Ferreira Ribeiro da Costa

E-mail: rodolfofrc@yahoo.com.br

José Raimundo Carvalho Júnior

E-mail: josecarv@ufc.br

Frederico Augusto Gomes de Alencar

E-mail: fredericoalencar91@gmail.com

Submetido em: 21/08/2019

Revisado em: $26 / 02 / 2020$

Aprovado em: 16/07/2020 